\title{
The Phenomenon of Proton Dissolving in Vacuum and of Proton Condensation from Vacuum. Two Forms of Protons, Structure of Nuclei, Electrons and Atoms
}

\author{
Kristina Zubow ${ }^{2}$, Anatolij Zubow ${ }^{1}$, Viktor A. Zubow* \\ ${ }^{1}$ Department of Computer Science, Humboldt University Berlin, Johann von Neumann Haus, Berlin, Germany \\ ${ }^{2}$ A IST Handels- und Consulting GmbH, Department of Research \& Development, Groß Gievitz, Germany \\ E-mail:zubow@informatik.hu-berlin.de,aist@zubow.de \\ Received April 18, 2010; revised May 15, 2010; accepted May 22, 2010
}

\begin{abstract}
It was investigated how react molecular clusters in water, starch, bio-matrices, polymers and in quartz on gravitation radiation from planets. Gravitation radiation (GR) was found to influence the proton jumping in hydrogen bonds that stabilize the cluster structure. There was given a method calculating parameters of GR as well as a mechanism of its resonance interaction with weak GR from molecular matter (WGR). WGR has been defined as the result of proton dissolving in vacuum connected with its simultaneous condensation in the nearest free space. Both dissolving and condensation proceed with super light velocity. The gravitation wave length has been determined experimentally and it depends on the planet masses (between Earth and Sun $\lambda \geq 62 \mathrm{~km}$, between Earth and Milky Way center $\lambda \geq 330 \mathrm{~km}$ ). GR has been characterized with super light velocity. After analyzing the Sun influence on water two forms of protons were found: in a condensed and dissolved state. A new model for the atomic nucleus has been suggested according to which the protons in the nucleus oscillate between condensed and dissolved state, where in the case of isotopes this state is partially destroyed. The models for $\mathrm{H}_{2}$ and Be shall be given. Electron orbitals in atoms and molecules were found to be caused by a stationary front of shock waves from condensing protons.
\end{abstract}

Keywords: Gravitation Waves, Proton, Dissolving, Vacuum, Planets, Electron, Form of Matter

\section{Introduction}

The proton jumping via hydrogen bridges (H-bonds) or via even a group of atoms in a molecule has been illustrated as follows:<smiles>CC(C)O[Hg]OC(C)C</smiles>
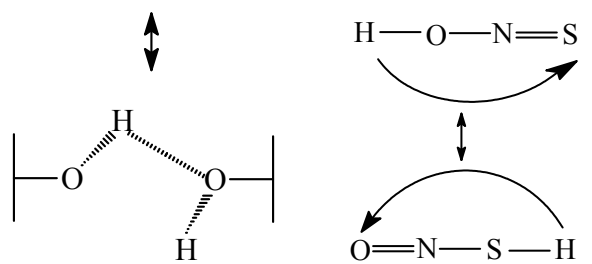

Scheme 1

The hydrogen atom that is linked with an oxygen atom builds simultaneously a H-bond $(\sim 0.2 \mathrm{~nm})$ with an other molecule fragment or an other molecule and it is able to jump to the second oxygen atom. Instead of oxygen other atoms like nitrogen; sulfur etc. can be applied to this scheme. Inner molecular jumps in low molecular substances can proceed at distances longer than $0.2 \mathrm{~nm}$ but in high molecular ones the talk is even on umpteen $\mathrm{nm}$.

This effect has been observed for many organic and inorganic substances and it is well described [1-3] though a proton jumping through electron orbitals of atoms and molecules seems to be more or less unprobably. Furthermore, because of unavailable mechanism models and analysis methods it is difficult to understand this effect. On the other side hydrogen bridges were found important for the stabilisation of the conformation of synthetic macromolecules and biomolecule coils (proteins, starch). Destruction of hydrogen bridges leads to a change of the conformation of protein macromolecules, to changed directions of biochemical reactions and finally to another behaviour of the organisms. In our earlier publications we informed on fields of unknown nature that are present in moleculare systems with hydrogen bridges. For instance, in [4] we described the "wall effect": near the wall there 
is a special gravitation excitation (water, salt solutions), that seems to be connected with hydrogen bonds and proton jumping. We developed the idea about the proton jumping via the vacuum to the nearest energy vacancy place in space. According to this hypothesis the proton jumping is connected with acceleration and breaking of masses in the proton leading to weak gravitation radiation. To identify a gravitation wave in a real time it is necessary to shift "the interference of the gravitation pair (mutual compensation) at the time or to expand the events in the space. For that we have to look for the resonance of gravitation waves between micro and macro objects for example, in gravitation pairs - between clusters in the molecular matter and masses of planets. To proof this hypothesis we developed a gravitation mass spectrometer (GMS) by which help we registered molecular mass clusters as well as their interaction with the surroundings $[5,7]$.

It was the aim of the present work to understand the mechanism of proton jumping in molecular matter as well as to find out the reasons that cause unknown fields in it.

\section{Experimentally}

As investigation objects the following substances were chosen: water, agarose hydrogel (97 wt.\% water), salt solutions ( $\mathrm{NaCl}, 3.5 \mathrm{wt} . \%)$, starch, polyethylene, atactic polystyrene (aPS, average molecular mass of $15 \mathrm{kDa}$ ) as well as bio matrices of eggs and potato tubers. The GMS-sensor was placed directly in the bio-matrices, pressed (1-2 $\mathrm{kPa})$ to powders or films or installed in aged for 6 month liquids. The GMS method is ascribed in [5-8]. Masses and oscillation frequencies of clusters in the samples were calculated by the Zubow equations using the Zubow constant $6.4 \times 10^{-15} \mathrm{~N} / \mathrm{m}$ for biomatrices, water and $6.8 \times 10^{-15} \mathrm{~N} / \mathrm{m}$ for starch and $5.2 \times 10^{-15} \mathrm{~N} / \mathrm{m}$ for aPS. Samples were placed in an iron (grounded) box that was isolated from light, anthropogeneous noises and heat/mechanical fields. The box itself was in a building far from industrial centers. Some curves, reflecting the energy flow (sum of cleaned signals) that achieves the GMS-sensor in the samples between August the $15^{\text {th }}$ and $16^{\text {th }} 2008$, are shown in Figure 1. The base line (dotted line) was obtained as follows. After noises were registered by GMS-sensor in vacuum $\left(10^{-4} \mathrm{~N} / \mathrm{m}^{2}\right)$ the real signals (first) were extracted where an analogous procedure was done for the sample without vacuum (second). The real sample signals were calculated by subtracting the second signals from the first ones [6]. 265784 represents the sum of all signals in the mass interval up to 4.3 billion Da.

\section{Results and Discussion}

The existence of cluster ensembles in molecular matter showing both wave and corpuscle properties are undoubtedly [9]. Clusters oscillate and noise. These oscillations e.g. in liquids are similar to "structure transfusion" and it is connected with a constant virtual mass jumping in the dynamic balance between noise energy stabilizing clusters [10] and heat (kT), that destroy clusters.

In Figure 1, the planet influence on the energy of cluster ensembles in molecular matter shall be shown. As

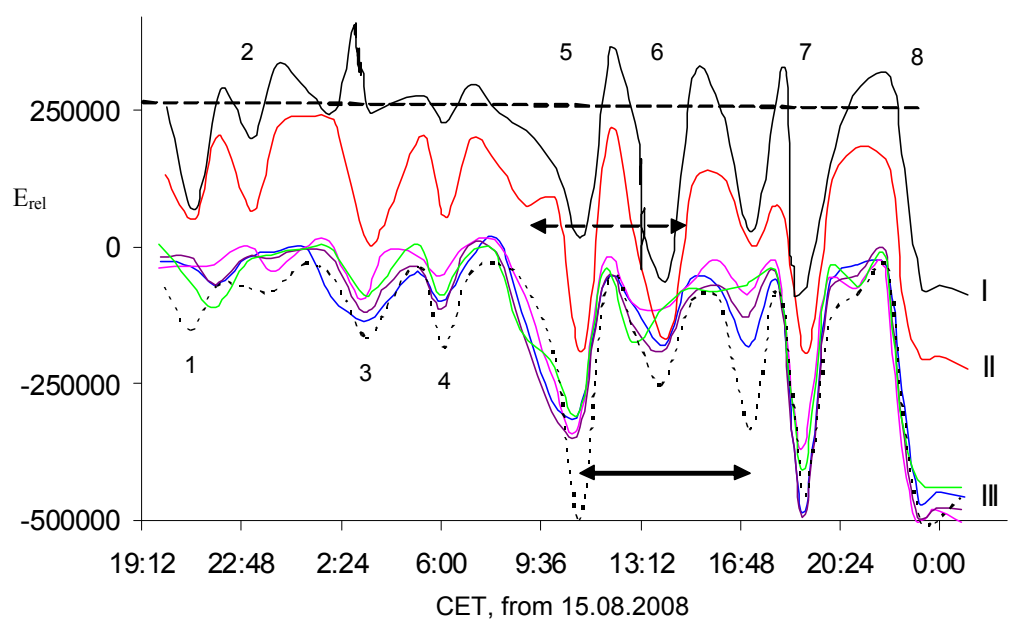

Figure 1. Energy flows in different substances: I-bio-matrix of fresh potato tuber, II-melted quartz (glass), III-water, methanol, bio-matrix of a hen's egg (egg white), polymers (dotted line), water solution of $3.5 \mathrm{wt}$.\% NaCl, $1-$ sundown, 2 -full Moon before the moon was covered by Earth $24 \mathrm{~h}$ later (8), 3-Uranus culmination, 4-Sunrise, 5-Mars culmination, 6-nearly simultaneous culmination of Mercury, Venus and Saturn, 7-Jupiter rise, 8-moon eclipse. By horizontal arrows the effects of interference of gravitation noises from Sun are given (dotted line: from 09:00 until 15:00) and Galaxy centre (dotted line: from 11:00 until 17:00). The curve is formed from points in which every point is a statistical value which isn't worse than $95 \%$. The base horizontal line corresponds to 265,784 relative units (state, on August $15^{\text {th }}, 2008$, at 19:12). 
visible the molecular cluster ensemble energy is sensitive to the planet constellation and this energy, for instance of gravitation nature, was found to be detectable by the GMS method.

Let us discuss two pairs of peaks at 09:00 and 15:00, and at 11:00 and 17:00, both peaks are on a line, that is perpendicularly to the line connecting the centers of the Sun and the Earth, and of the Milky Way and the Earth, accordingly. The peaks can be interpreted as interference of gravitation waves and with the help of the Junge equation the wave length can be calculated and compared with literature references. The calculation was made using

$$
\lambda \geq D \cdot S / R
$$

where $\lambda$ - average gravitation wave length, $R$ - distance from the gravitation source to sample (until Sun 150 mio $\mathrm{km}$, until Milky Way center $10,000 \mathrm{pc}), D$-distance between peaks, amounting to $D=2 \cdot r \cdot \sin \left(90^{\circ}-53^{\circ}\right) \cdot \sin$ $\left(120^{\circ} / 2\right), r=6370 \mathrm{~km}$ (radius of Earth), $S$ - diameter of source (Sun, 1,392,000 km, Galaxy center 500 pc), here the role of two slits played the Earth atmosphere and the role of display - area which connects the peaks with each other and stands vertically to the gravitation source. Thus, $\lambda \geq 62 \mathrm{~km}$ (gravitation waves of Sun) and $\lambda \geq 330 \mathrm{~km}$ (gravitation waves of the Galaxy center). The gravitation wave length calculated by Hickey [11] was found to be $\sim 100 \mathrm{~km}$ furthermore Smirnov described a device for the registration of gravitation excitation in the frequency interval $10 \mathrm{~Hz} . .40 \mathrm{kHz}$ [12], which includes all investtigated cluster oscillations in molecular matter. We can therefore notice with a great probability that the GMS device registers directed gravitation radiation (GR). Now we want to analyze how GR influences the long range order in water whose structure was already proved experimentally [8]. Inside the clusters, the water molecules are linked with each other by two hydrogen bridges but on the cluster surface the water molecules possess only one hydrogen bridge (HB) for their interaction with the surroundings. If the proton jumping in HB proceeds with emission of weak GR then we have to expect a resonance between weak GR of molecular matter and GR of planets. The proof of this assumption is given in Figure 2.

As visible from Figure 2 the number of clusters in water reacts on the planets' GR where this effect is shown most strongly at full moon (23:16, point M) and eclipse of the moon (the moon is covered to $80 \%$ by the Earth). The GR of Jupiter at its rise has a strong influence on the cluster number in water too. To understand the structure of GR we investigated GR of Jupiter (Figure 3). GR of Jupiter consists of two parts which were ascribed to signals of "gravitation Jupiter" and of "visible Jupiter". The

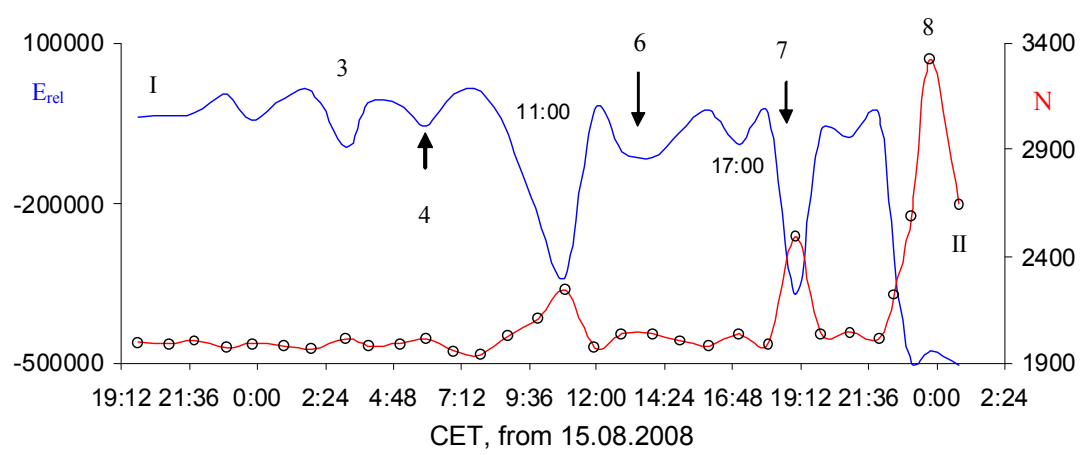

Figure 2. Behavior of the GR energy curve registered by GMS-sensor in water (I) and of the number of cluster kinds (II) in dependence on events in the near space, cluster ensembles up to 4.3 billion Da. Comments see in Figure 1.

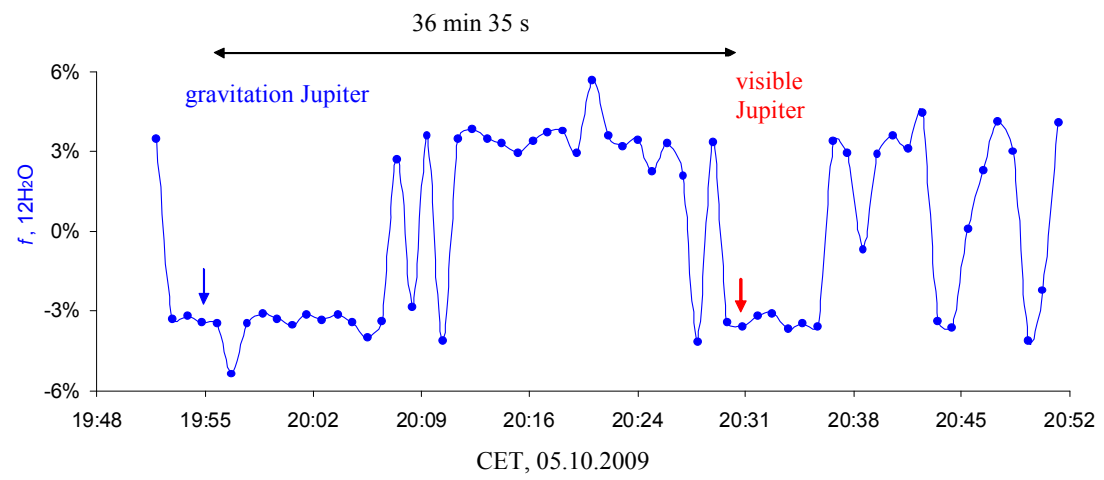

Figure 3.Signal intensities of the base water cluster in agarose hydrogel when the flows of both Jupiter gravitation energy kinds are in the slide plane of gravitation proton resonance (SPGPR). Distance to Jupiter-658,629,000 km; altitude $19.7^{\circ}$. SPGPR - plane going through the Earth rotating axis and the sample place on the Earth surface. 
sample was fully protected from visible light therefore, the signal of "visible Jupiter" can be interpreted as ripples of main Jupiter GR $[7,11]$. That means we have the right to use the correlation between the visible and gravitation constellation of planets.

In Figure 4, computer models for the simplest water clusters, consisting of the base water cluster $\left(\mathrm{H}_{2} \mathrm{O}\right)_{11 \pm 1}$ $[13,14]$, and experimentally observed methanol clusters are given [8]. The base water cluster belongs to an ensemble of dominating clusters which were formed forced in the white noises: $\left(\mathrm{H}_{2} \mathrm{O}\right)_{11 \pm 1},\left(\mathrm{H}_{2} \mathrm{O}\right)_{100},\left(\mathrm{H}_{2} \mathrm{O}\right)_{178},\left(\mathrm{H}_{2} \mathrm{O}\right)_{280}$, $\left(\mathrm{H}_{2} \mathrm{O}\right)_{545},\left(\mathrm{H}_{2} \mathrm{O}\right)_{903},\left(\mathrm{H}_{2} \mathrm{O}\right)_{1351},\left(\mathrm{H}_{2} \mathrm{O}\right)_{1601},\left(\mathrm{H}_{2} \mathrm{O}\right)_{1889}$ etc.. Jupiter at rise (Figure 1) and culmination (Figure 4) strongly influences by its gravitation radiation the long range order in water and destroys the cluster ensemble temporarily. On the other side, the destruction of the water cluster ensemble has been observed during the gravitation interference (signal at 11:00) and even at GR of Uranus and sunrise (Figure 2).

Cluster ensembles in methanol react analogously to GR flow of planets where the highest destruction of hydrogen bridges takes place at Jupiter rise. However, the behavior of polyethylene, which doesn't have any hydrogen bridges, is something differently the strongest cluster ensemble destruction is at Moon eclipse and at GR interference of the Galaxy center and WGR of polyethylene (11:00 and 17:00).

In the Table 1, the GR frequencies of planets and water molecular clusters that were calculated by the Zubow equation are given.

Let us now discuss the hydrogen bridges (HB) in the amorphous part of starch (Figure 5). As shown in Figure $5 \mathrm{HB}$, reflecting conformation changes in the starch coils, react on GR of planets. In Figure 6, the review GM-spectra of starch powder at Jupiter rise will be given (see Figure 1 too), the destruction of the hydrogen bridges that form the globular structure of this polymer can be seen. This destruction process proceeds reversibly and is an indication for a special resonance interaction between gravitation radiations. On the other side the dominating influence of planets' GR on a cluster ensemble in molecular matter often leads to a simultaneous energy inversion of the density of all clusters. In starch, the conformation of macromolecule coils are known to be stabilized by HB and its destruction, which is caused by resonance with planets' GR, should be modelable by a forced compression. At pressure the length of HB shall be changed and the oxygen influence on the "naked" proton becomes stronger. We found, that by pressing the starch powder mechanically $(1 \ldots 5 \mathrm{kPa})$ the hydrogen bonds were forced and reversibly destroyed too conesquently, mechanical pressure is comparable with planets' GR.

Thus, the existence of weak gravitation radiation in molecular matter, which is caused by super light mass transfer in hydrogen bridges, has been concluded. It is hard to imagine this process, as a simple jump on a dishard to imagine this process, as a simple jump on a dis-

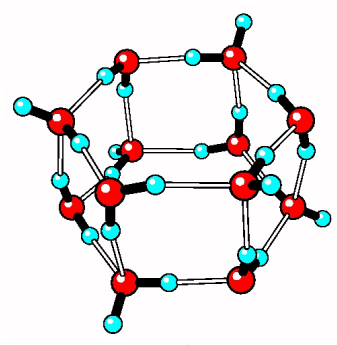

$\left(\mathrm{H}_{2} \mathrm{O}\right)_{12}$

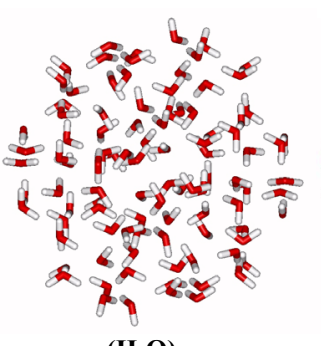

$\left(\mathbf{H}_{2} \mathbf{O}\right)_{100}$

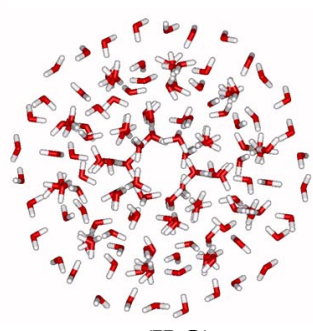

$\left(\mathrm{H}_{2} \mathrm{O}\right)_{160}$

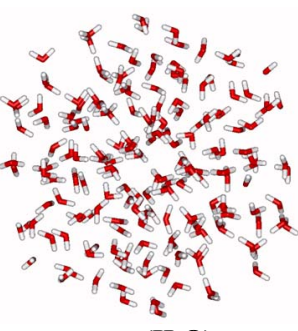

$\left(\mathrm{H}_{2} \mathrm{O}\right)_{184}$

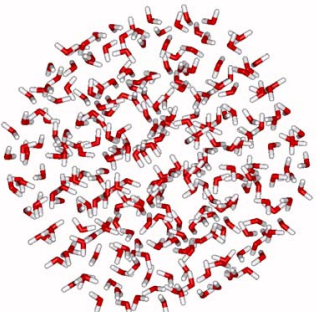

$\left(\mathrm{H}_{2} \mathrm{O}\right)_{280}$

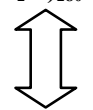

Expanded structure (ES) Collapseded structure (CS)

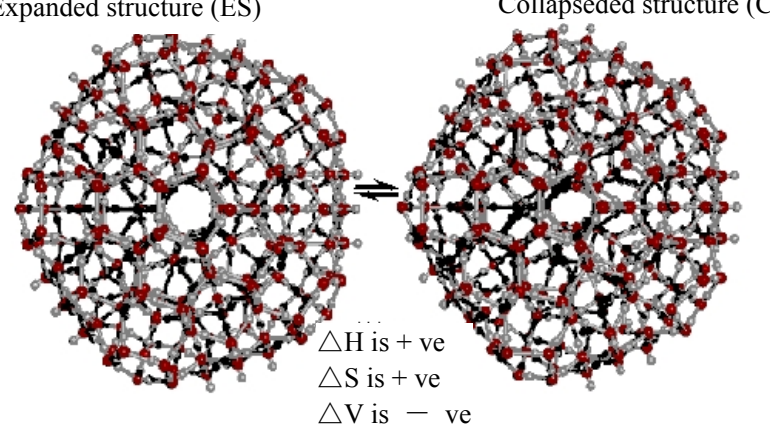

Figure 4. Calculated water cluster models (with kind permission of the professors Chaplin (http://www.lsbu.ac.uk/water/inde x.html) and Lenz [14]) and methanol clusters, experimentally observed by the authors [8]. 


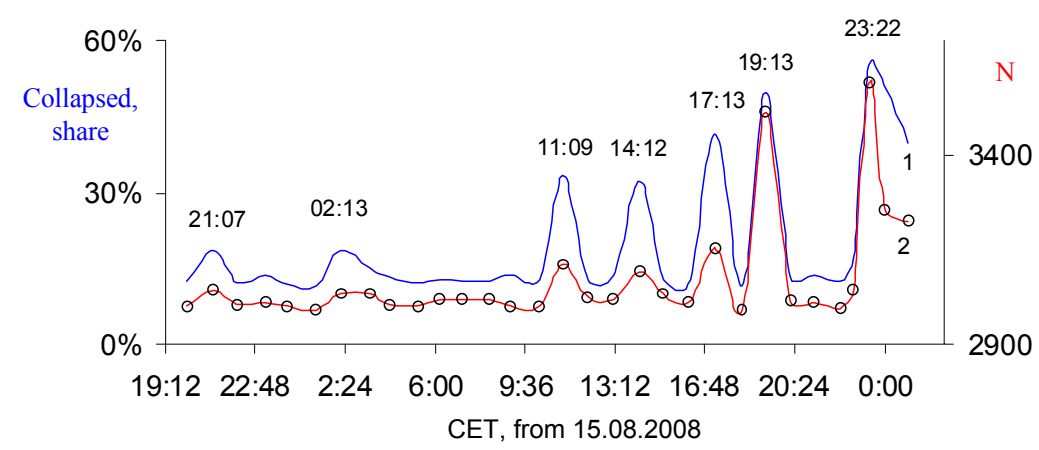

Figure 5. Share of collapsed clusters (1) and of cluster kinds' numbers (2) in the amorphous part of potato starch observed at GR influence of planets (Figure 1).

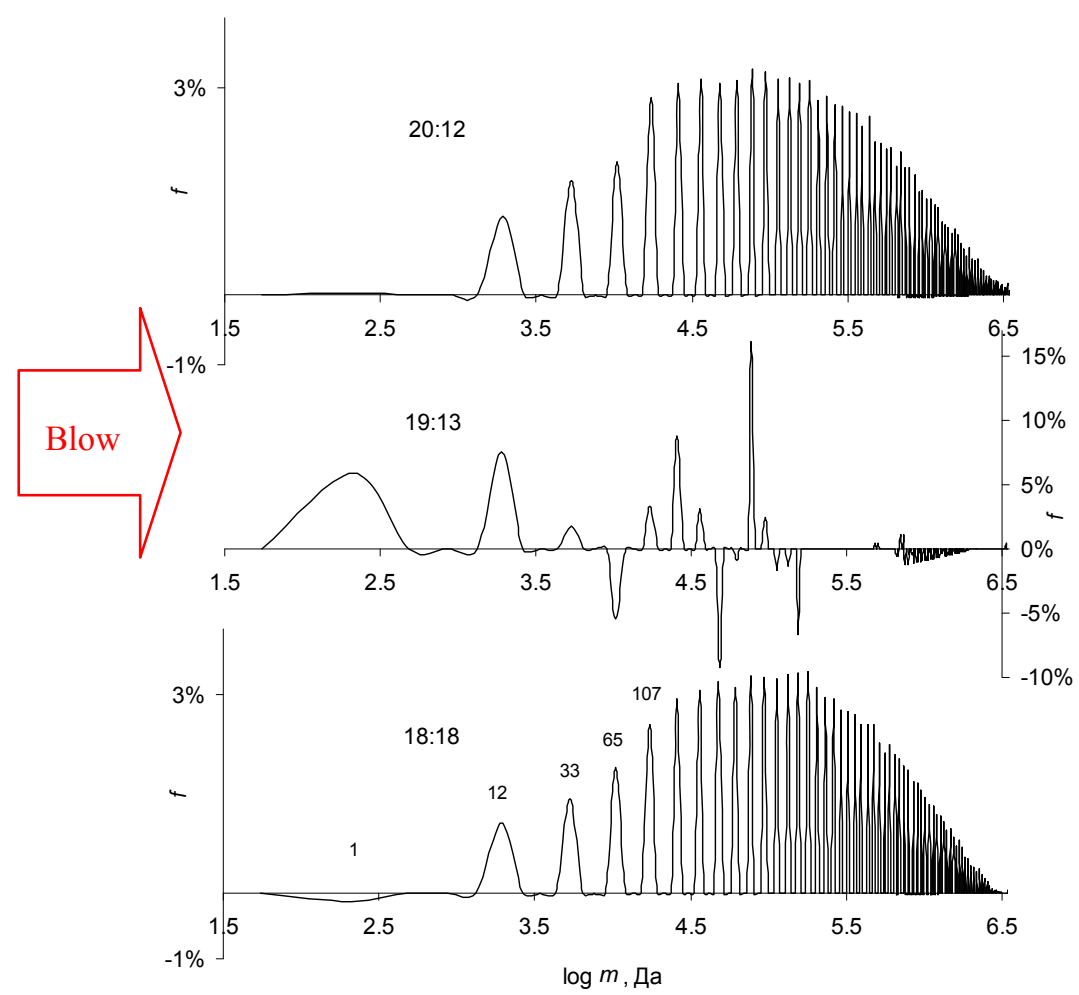

Figure 6. FNS of potato starch (powder $1.55 \mathrm{mmol}, 0.5 \mathrm{~cm}^{3}$ ) at Jupiter rise (Figure 5). Weak shock wave $\left(p<1 \mathrm{~N} / \mathrm{m}^{2}\right), \mathrm{Zubow}$ constant is equal to $6.8 \times 10^{-15} \mathrm{~N} / \mathrm{m}$. The numbers over the signals correspond to the number of $\alpha$-D-glycopyranose in a cluster. The number of cluster kinds (signals) in the investigated mass ensemble amounts to: 102 at 18:18, 59 at $19: 13$ and 104 at 20:12 (Central European Time, 16.08.2008). The distance between Jupiter and Earth is $655 \times 10^{6} \mathrm{~km}$.

Table 1. The main noise frequencies of planets and of the sun, that were calculated according to $m=N_{\mathrm{A}} \cdot 10^{11} \cdot \omega^{-2}$, where compared with the frequencies of water clusters in a potato bio-matrix, $m=10^{11} \cdot \omega^{-2}$.

\begin{tabular}{ccccc}
\hline & Characteristics & \multicolumn{2}{c}{ Molecule clusters* } \\
\hline Planet & $\boldsymbol{m}, \mathbf{g}$ & $\mathbf{\omega , ~ H z}$ & $\mathbf{\omega , ~ H z}$ & Formula \\
Mercury & $3.40 \mathrm{E}+26$ & $13309 \pm 2000$ & $11111 \pm 2000$ & $\left(\mathrm{H}_{2} \mathrm{O}\right)_{45}$ \\
Venus & $4.87 \mathrm{E}+27$ & $3517 \pm 200$ & $3431 \pm 200$ & $\left(\mathrm{H}_{2} \mathrm{O}\right)_{472}$ \\
Earth & $5.97 \mathrm{E}+27$ & $3176 \pm 200$ & $3193 \pm 200$ & $\left(\mathrm{H}_{2} \mathrm{O}\right)_{545}$ \\
Mars & $6.40 \mathrm{E}+26$ & $9700 \pm 1500$ & $7454 \pm 1500$ & $\left(\mathrm{H}_{2} \mathrm{O}\right)_{100}$ \\
Jupiter & $1.90 \mathrm{E}+30$ & $178 \pm 20$ & $178 \pm 20$ & $\left(\mathrm{H}_{2} \mathrm{O}\right)_{174420}$ \\
Saturn & $5.69 \mathrm{E}+29$ & $325 \pm 30$ & $333 \pm 30$ & $\left(\mathrm{H}_{2} \mathrm{O}\right)_{50124}$ \\
Uranus & $8.70 \mathrm{E}+28$ & $832 \pm 50$ & $827 \pm 50$ & $\left(\mathrm{H}_{2} \mathrm{O}\right)_{8131}$ \\
Neptune & $1.03 \mathrm{E}+29$ & $765 \pm 40$ & $769 \pm 40$ & $\left(\mathrm{H}_{2} \mathrm{O}\right)_{9385}$ \\
Moon & $7.35 \mathrm{E}+25$ & $28624 \pm 4000$ & $21517 \pm 4000$ & $\left(\mathrm{H}_{2} \mathrm{O}\right)_{11 \pm 1}$ \\
Sun & $1.99 \mathrm{E}+33$ & $5.5 \pm 1$ & $5.5 \pm 1$ & $\left(\mathrm{H}_{2} \mathrm{O}\right)_{36453036}$ \\
\hline
\end{tabular}


tance of $0.2 \mathrm{~nm}$ or longer, a process in which the proton must penetrate a "net" from electron clouds of atoms and molecules. However, there are another way, namely the transition of the proton into the vacuum (dissolving) connected with the arising of his equivalent simultaneously in the next free energy place. This process of super fast dissolving and condensation of mass could cause WGR from molecular matter [15]. Under the examined substances stand out the ones, those don't have any HB (aPS, polyethylene). The proton jump in HB is therefore a special case of the general phenomenon for the proton jump from the atomic nucleus into vacuum and reversed.

To evaluate the velocity of the proton jumping in vacuum the results of GR wave length analysis were applied (Figure 1) using in the first approximation the Planck equation $E=h \cdot c_{\Gamma} \cdot \lambda$, where $h$-Planck constant, $\lambda$-wave length, $\mathrm{m}, c_{\Gamma}-$ velocity, $\mathrm{m} / \mathrm{s}$. The energy value (E) can be considered as the disintegration energy of starch coils [16]. We assume that this value amounts to $\sim 2 \mathrm{~kJ} /$ mole, then

$$
c_{\Gamma}=E \cdot \lambda / h>15 \times 10^{35} \mathrm{~m} / \mathrm{s} .
$$

This velocity is $5 \times 10^{27}$ times higher than the light velocity and the GR frequency is equal to $c_{\Gamma} \cdot 1 / \lambda=2.32$ $\times 10^{31} \mathrm{~s}^{-1}$ which doesn't agree with the gravitation frequencies published in [17-20] as well as with the conclusions on the GR influence on the long range order in molecular matter. However, this situation can be cleared with the results of Kokkotas [21] according to whom at moving of masses their gravitation waves initiate ripples of smaller ones (gravitation noise), that spread with light velocity. The gravitation noise we observed to be the reason for the forced oscillation of clusters in molecular matter and matter in space but the clusters themselves are the result of stationary gravitation waves. Then, the energy dependence of the main gravitation waves on their wave length can be given as:

$$
E=z \cdot \lambda,
$$

where $z \sim(2 \ldots 3) \cdot 10^{-2} \mathrm{~J} / \mathrm{m}, \lambda \sim 65,000 \ldots 100,000 \mathrm{~m}$.

Of course, the proton moving with super light velocity has to understand as a short living time at the level of proton dissolving in vacuum $\left(P_{\mathrm{p}}\right)$ and proton condensation from vacuum $\left(P_{\mathrm{K}}\right)$. Then, the model for the proton jumping can be shown as follows.

In this scheme, the distance between dissolving and

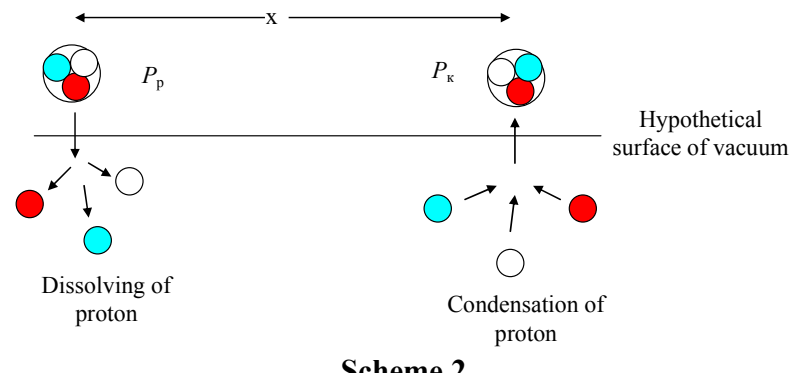

Scheme 2 condensing proton can be forced changed for example, by a simple sample compressing [7] or by the resonance with analogous processes from planets.

This nucleus model doesn't contradict the idea about the cluster formation in boson matter, according to which the mass transformation proceeds to strictly deterministic gravitation laws [7]. The physicists have unbelievable done, to explain the stability of great and thick accumulations of positively charged particles in a very small volume of the nucleus. One tried to find a force which holds these particles together however it wasn't found. Now as we understand the proton as an oscillating substance (dissolving and condensing) the nucleus formation of chemical elements and their isotopes should be clearer. In nuclei, the proton dissolving proceeds in the energy balance with the condensation of the neighboring proton (see scheme). In this case, the probability that two positive charges are besides each other, gets lower but the gravitation interaction in the nucleus becomes more dominant.

The instability of radioactive isotopes has been explained by the destruction of the oscillation harmony in the nucleus. Radioactive isotopes are characterized by a high potential energy of their nuclei (as a pendulum system) and its minimization is connected with nucleus disintegration.

The molecule stability of hydrogen $(\mathrm{H}-\mathrm{H})$ can be better explained by this model according to which the protons alternately dissolve and condense and the electrons permanently catch up with the positive charges (condensed proton). Here the electrons permanently rotate around the condensed proton and its Fata Morgana "(dissolved proton) leading to multiple canonical structures for instance $\mathrm{H}-\mathrm{H}$.

For the beryllium nucleus, two canonical structures shall be shown, for example (Figure 7) this scheme is analogous to the one which describes the formation of clusters in molecular matter [9].

The electron clouds around the nuclei can be understood as stationary shock waves arose from condensing protons from vacuum. The shock wave front is in balance with the energy state of the vacuum $1.4 \times 10^{16} \mathrm{~kJ} / \mathrm{m}^{3}[7,9]$. This front (electron orbital) is permanently generated by high frequency shock waves from the condensing proton and at proton dissolution; it doesn't manage to dissipate in vacuum and saves therefore its spherical form (model for hydrogen atom, Figure 8) because of a higher inertia of the dissipation process, probably. A proof for this model could be that the electron orbital in the hydrogen atom oscillates (known as electron transition from one energy state to the other one). In accordance with this logic the energy released at proton condensation should correspond with the vacuum energy namely with $\sim 1.4 \times$ $10^{16} \mathrm{~kJ} / \mathrm{m}^{3}$.

For multiple proton nuclei, the spherical shock wave construction shall be changed to differently formed ones, 


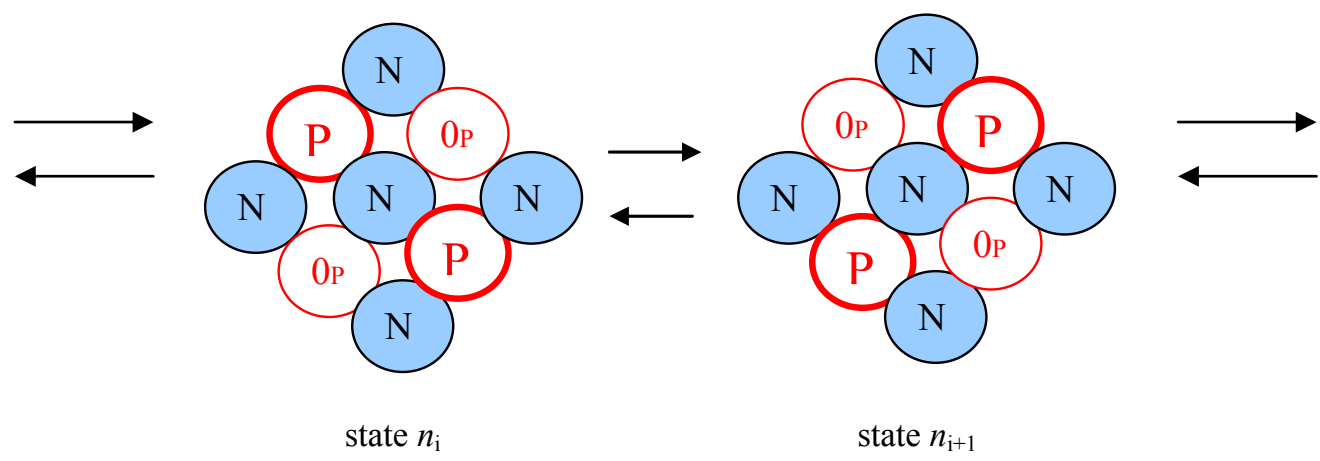

Figure 7. Statistical model for the nucleus formation of beryllium (4 protons $(\mathrm{P})$ and 5 neutrons $(\mathrm{N}))$ ). $\mathrm{P}$-proton condensed from vacuum, $0 \mathrm{p}$ - place of the dissolved proton in vacuum. The most probable states are shown.
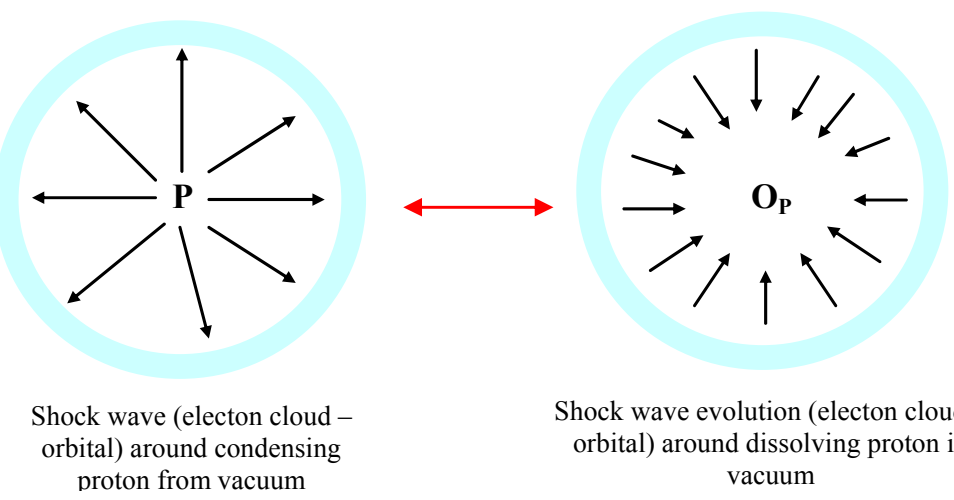

Shock wave evolution (electon cloud orbital) around dissolving proton in vacuum

Figure 8. Model for the formation of a spherical electron orbital in the hydrogen atom. This orbital is caused by a shock wave front which arises from the pulsating of the condensing proton from the vacuum (left) and from the front movement at proton dissolving in vacuum (right). The structure and energy of the vacuum are described in [7,9].

known as orbital hybridization in chemistry. Here the hybridization reflects an energy minimization process. On the other side the state of free electrons can be understood as an energy cluster of excited matter from vacuum that exists in the movement form only.

According to this model of nucleus, the molecule is less caused by the unification of nuclei through electron orbitals than by a forced cluster formation of protons in stationary gravitation waves of the space [7]. The concentration of atomic nuclei in a cluster represents a molecule but that one of any molecules a molecule cluster. Starting out from this model the electron orbital can be imaginated as a shock wave front in the compressed vacuum being another form of the matter. Under special conditions the shock wave front could be concentrated in a cluster of negative energy-electron-particle. We therefore have to work with two matter forms which aren't mixable with each other, vacuum matter and matter that shall be released or absorbed at condensation/dissolution of a proton.

The GM-spectra observations of water at the period when the Sun is in SPGPR could be an additional proof for the dissolving of the proton and for its existence in two forms (dissolved and condensed). The results of these observations shall be given in Figure 9.

As visible from Figure 9, all base skeletal water clusters are present in water of the agarose hydrogel [22]. The GM-spectra are identical up to the entrance of the Sun in SPGPR (13:14) and after its exit from SPGPR (13:18). The molecular clusters are given in their expanded forms indicating a strong inter- cluster interaction through hydrogen bonds. In these bonds, the, by the Paulings so-called, "naked" protons [23] are in the condensed state. At the entrance of the Sun in SPGPR (13:15) the protons disappear, to be explained with their forced dissolution in physical vacuum and the remove to the Sun [7].

Thus, the structure of hydrogen bonds between molecular clusters has been destroyed that means the inter-cluster hydrogen bonds possess the weakest protons that shall be removed to the Sun center first. In the following two minutes in the long-range order of water, an other form of hydrogen bonds was observed where water clusters became collapsed $(13: 16 \ldots 13: 17)$. A weakened proton is present here in the inter-cluster hydrogen bonds, that return into the normal condition at 13:18 again, the same state before the entrance of the Sun in SPGPR. Therefore, two forms of "naked" protons and a state with- 

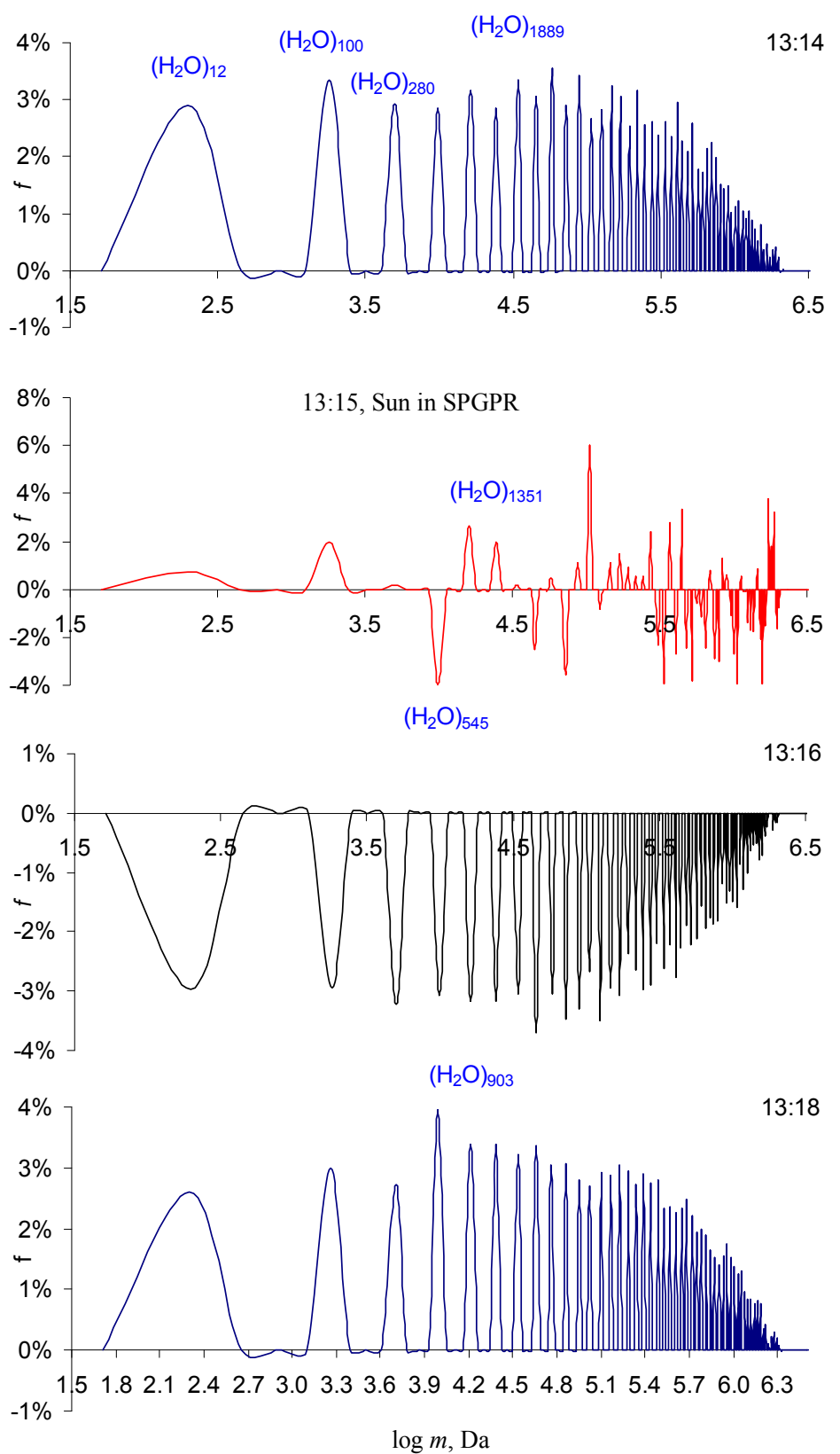

Figure 9. Dynamics of the proton state in hydrogen bonds between water clusters in agarose hydrogel at the period when the Sun is in SPGPR (August $7^{\text {th }}$ 2009).

out any protons were concluded to be present in hydrogen bonds (Figure 10).

At this time, to differentiate between the dissolution and condensation state of protons is impossible because they proceed with a super light velocity however, the talk can be on canonical structures in their harmonic resonance. It doesn't have to be excluded that neutrons take place in this family of pendula too. The gravitation strength which holds the nucleus as a unity together is the result of the minimization of the potential energy of this mass ensemble [7].
As shown in Figure 10 the proton dissolving is accompanied by the disappearance of its electron (stationary front of shock wave). Here in the matter, only one free radical of the so-called "free electron", as it was understood by the nature scientists of the 20th century, remains. If only the proton should dissolve, then the matter should have a gigantic negative charge what isn't the case, however. This simple logic supports the suggested electron model as phenomenon of stationary shock wave front (Figure 8). Our universe consists consequently of two not with each other mixable, highly dis- 
$13: 14$<smiles>CO[Hg]OC</smiles>

$13: 15$

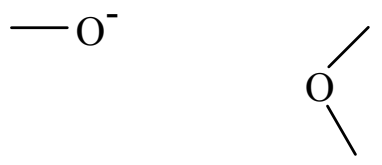

$13: 16$

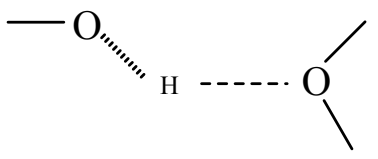

$13: 18$

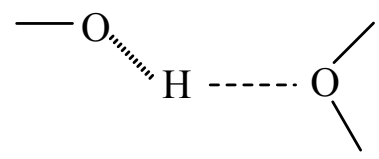

Scheme 2

Figure 10. Scheme illustrating the inter-molecular clusters states of hydrogen bonds when the Sun hits SPGPR.

persed and competitive forms of the matter: mater of vacuum and matter of proton.

\section{Conclusions}

Thus, the mass transfer of protons of hydrogen bonds or atomic nuclei in vacuum (dissolution) proceeding with a super light velocity as well as the almost simultaneous appearance of protons from vacuum (condensation) at the nearest vacant place of the space were concluded to be the reason for weak gravitation radiation of molecular matter. The structure of the hydrogen atom reflects the surface state between two forms of matter: matter in vacuum and matter consisting of dissolved protons. These two forms could exist independently of each other. The electron orbitals in atoms and molecules are stationary shock waves in vacuum coming from two fast running processes (dissolving/condensing of protons) in atomic nuclei.

\section{Acknowledgements}

The authors gratefully acknowledge the Aist Handelsund Consulting $\mathrm{GmbH}$ for financial support, the group of astrophysicists of professor Vasiljev S. A. (Greece) and professor Smirnov V. N. (Russia) for the fruitful discussion.

\section{References}

[1] A. N. Isaev, "Co-Operation Interactions of Hydrogen Bonds in the Processes with Proton Jumping and Water
Molecules Participation. Modelling of Biochemical Systems", Russian Journal of Chemistry, Vol. LI, No. 5, 2007, pp. 34-48.

[2] D. F. Baisa, E. D. Chesnokov, A. I. Ovcharenko and I. G. Vertegel, "NMR Study of Proton Dynamics in Ferroelectric KIO32HIO3 Crystal," Functional Materials, Vol. 15, No.1, 2008, pp. 19-22.

[3] P. Jaque, A. Toro-Labbe, P. Politzer and P. Geerlings, "Reaction Force Constant and Projected Force Constants of Vibrational Modes along the Path of an Intramolecular Proton Transfer Reaction," Chemical Physics Letters, Vol. 456, No. 4-6, 2008, pp. 135-140.

[4] A. V. Zubow, K. V. Zubow and V. A. Zubow, "The Phenomenon of Water Clusters Distribution in Water and Solvated Clusters of Ion Pairs of $\mathrm{NaCl}$ in Solution Near the Wall, the Wall Effect," Journal of Chemical Industry, Vol. 83, No. 6, 2006, pp 300-306.

[5] K. V. Zubow, A. V. Zubow and V. A. Zubow, "Using of Flicker Noise Spectroscopy for Non Destroyng Analysis of Nano Structures," Zavodskaja Laboratorija. Diagnostics of Materials, Vol. 74, No. 9, 2008, pp. 40-45

[6] K. V. Zubow, A. V. Zubow and V. A. Zubow, "The Dynamics of Low Frequency Movements of Molecular Clusters in the Hardening Process of Epoxide Resins," Chem Promislennost Segodnja, No. 9, 2008, pp. 12-21.

[7] K. V. Zubow, A. V. Zubow and V. A. Zubow, "Principles of Gravitation Stectroscopy. New Form of Molecular Matter Processes Fields," Aist H \& C gmbH, Berlin, 2010, electronic book, www.zubow.de

[8] K. V. Zubow, A. V. Zubow and V. A. Zubow, "Cluster Structure of Liquid Alcohols, Water and N-Hexane," Journal of Applied Spectroscopy, Vol. 72, No. 3, 2005, pp. 321-328.

[9] K. V. Zubow, A. V. Zubow and V. A. Zubow, "Ensemble of Clusters-New Form of Molecular Matter, Risks and Chances. Zubow Equations," In: F. Columbus, Ed., Chemical Industry Challenges Issues and Prospects, Nova Science Publishers, NewYork, 2010.

[10] W. Horsthemke and R. Lefever, "Noise-Induced Transitions. Theory and Applications in Physics, Chemistry and Biology. Edit. Hermann Haken," Springer-Verlag, Berlin, Heidelberg, Tokio, 1984.

[11] Hickey, P. Michael and Y. H. Yu, "A full-Wave Investigation of the Use of a "Cancellation Factor" in Gravity Wave-OH Airglow Interaction Studies," Journal of Geophysical Research, Vol. 110, No. 1, 2005, pp. 1-15.

[12] V. N. Smirnov, N. V. Egorov and I. S. Shchedrin, "A New Detector for Pertrubations in Gravitational Field," Progress in Physics, Vol. 2, No. 4, April 2008, pp. 129133.

[13] E. V. Bogdanov and G. M. Manturova, "Equiclaster Model of Water," Biomedicinskaja Radioelektronika, No. 7, 2000, pp. 19-28.

[14] A. Lenz and L. Ojamäe, "On the Stability of Dence Versus Cage-Shaped Water Clusters: Quantum-Chemical Investigations of Zero-Point Energies, Free Energies, BasisSet Effects and IR Spectra of $\left(\mathrm{H}_{2} \mathrm{O}\right)_{12}$ and $\left(\mathrm{H}_{2} \mathrm{O}\right)_{20}$," 
Chemical Physics Letters, Vol. 418, 2006, pp. 361-367.

[15] "Fisitcheskij Encikloped. Slowar," Moscov, Sovietskaja Enciclopedija, 1984, p. 137.

[16] S. S. Kozlov, A. V. Krivandin, O. V. Shatalova, T. Noda, E. Bertoft, J. Fornal and V. P. Yuryev, "Structure of Starches Extracted from Near-Isogenic Wheat Lines," Journal of Thermal Analysis and Calorimetry, Vol. 87, No. 2, 2007, pp. 575-584.

[17] A. Di Virgilio, S. Bigotta, L. Barsotti, et al., "Experimental Upper Limit on the Estimated Thermal Noise at Low Frequencies in a Gravitational Wave Detector," $P h$ ysical Review D: Particles, Fields, Gravitation, and Cosmology, Vol. 76, No. 12, 2007, pp. 122004/1-122004/10.

[18] E. Katsavounidis and S. Ballmer, "For How Long will Gravitational Waves Remain Hidden?" Physics Letters A, Vol. 347, No. 1-3, 2005, pp. 33-37.

[19] R. T. Stebbins and P. L. Bender, "A Lunar Gravitational
Wave Antenna Using a Laser Interferometer," AIP Conference Proceedings, Vol. 202. 1990, pp. 188-201.

[20] G.-R. Dorota and L. Francois, "The Final Phase of Inspiral of Strange Quark Star Binaries General Relativity and Quantum Cosmology," Preprint Archive, (Los Alamos National Laboratory, 2008, arXiv: 0801.4829v1), pp.1-13. http://aps.arxiv.org/PS_cache/arxiv/pdf/0801/0801.4829v 1.pdf

[21] D. K. Kokkotas, "Gravitational Waves," Acta Physica Polonica, Vol. 38, No. 12, 2007, pp. 3891-3923.

[22] A. V. Zubow, K. V. Zubow and V. A. Zubow, "Investigation of the Distribution of Water Clusters in Vegetables, Fruits and Natural Waters by Flicker Noise Spectroscopy," Biofizika, Vol. 52, No. 4, 2007, pp. 585-592.

[23] L. Pauling and P. Pauling, "Chemistry," W. H. Freeman and Company, San Francisco, 1975. 